\title{
Is Exchange Transfusion for Severe Neonatal Infection Preferable to Polymyxin B-immobilized Fiber Column for Direct Hemoperfusion: Pros and Cons
}

\author{
Naoto Nishizaki ${ }^{1}$ and Toshiaki Shimizu ${ }^{2}$ \\ ${ }^{1}$ Department of Pediatrics, Juntendo University Urayasu Hospital, Urayasu, Chiba, Japan \\ ${ }^{2}$ Department of Pediatrics, Juntendo University Graduate School of Medicine, Tokyo, Japan
}

Tohoku J. Exp. Med., 2021 June, 254 (2), 141-142.

\section{Dear the Editor,}

As we perused with great interest the article "Cytokine Profiles Before and After Exchange Transfusions in Severe Late-Onset Neonatal Group B Streptococcus Meningitis: A Case Report" by Chishiki et al. (2021), we deemed it necessary to reappraise their viewpoints regarding the strategies for severe neonatal infection. In their case, an 11-day-old infant received antibiotics and underwent two exchange transfusions (ExTs) for group B streptococcus (GBS) meningitis. Although the change in serum IL-6 level before and after ExTs was from $86,900 \mathrm{pg} / \mathrm{mL}$ to $3,299 \mathrm{pg} / \mathrm{mL}$ (decrease rate, 96.2\%), ExTs did not improve her neurological prognosis. Despite ExTs could be a feasible therapy for infection with hypercytokinemia, they concluded that establishing a preventive strategy is more important. We concur with their conclusion; however, an issue in the therapeutic strategies needs to be considered.

We herein would like to show the reliability of polymyxin B-immobilized fiber column for direct hemoperfusion (PMX-DHP) for neonatal GBS infection. A male infant with gestational age of 25 weeks and birthweight of $788 \mathrm{~g}$ was delivered via cesarean section to a 34-year-old woman because of fever caused by chorioamnionitis. Three hours after birth, he was diagnosed with septic shock in accordance with Goldstein's criteria (Goldstein et al. 2005). The cardiopulmonary status rapidly deteriorated, and serum IL-6 level was $6,792 \mathrm{pg} / \mathrm{mL}$, measured using RAY-FAST® (Toray, Tokyo, Japan). Therefore, we diagnosed hypercytokinemia provoked by septic shock, and cytokine/mediator removal using PMX-DHP was performed in addition to antibiotic administration. The PMX-DHP procedure was similar to those utilized in a previous study (Nishizaki et al.
2016). PMX-DHP was conducted for a total time of $5.2 \mathrm{~h}$. Then, the patient was withdrawn from shock status, and the change in IL-6 level after PMX-DHP was $73 \mathrm{pg} / \mathrm{mL}$ (decrease rate, 98.9\%). The following day, GBS was cultured from the blood. Presently, he could walk and speak words, even though he had periventricular leukomalacia at four years of age.

Table 1 shows a comparison of the patient's characteristics and clinical course of both cases. Although the neurological prognosis cannot be compared only by changes in IL-6 level and the presence or absence of meningitis, we emphasize that PMX-DHP is more suitable than ExTs. In PMX-DHP, which is performed by extracorporeal circulation using a closed circuit, the antibiotics are never lost from the patient's blood. Conversely, in ExTs, antibiotics and blood components are excreted outside the body. Thus, it should never be forgotten that the gold standard treatment of infection is sufficient blood level of antibiotics. Although PMX-DHP is originally effective against endotoxinemia of Gram-negative bacteria and there is no guarantee of effective treatment against Gram-positive coccus (e.g., GBS), we believe that PMX-DHP has both endotoxin and cytokine removal effects (Nishizaki et al. 2017, 2020). As a limitation, no difference in the therapeutic effect of ExTs and PMX-DHP on infection with hypercytokinemia has been reported even in neonates. Therefore, further studies are required to evaluate optimal strategy in addition to the efficacy of cytokine/mediator removal therapy for neonates with severe bacterial infection.

\section{Conflict of Interest}

The authors declare no conflict of interest.

Received May 8, 2021; accepted May 10, 2021. Published online July 1, 2021; doi: 10.1620/tjem.254.141.

Correspondence: Naoto Nishizaki, M.D., Ph.D., Department of Pediatrics, Juntendo University Urayasu Hospital, 2-1-1 Tomioka, Urayasu, Chiba 279-0021, Japan.

e-mail: nishizak@juntendo.ac.jp

(C)2021 Tohoku University Medical Press. This is an open-access article distributed under the terms of the Creative Commons Attribution-NonCommercial-NoDerivatives 4.0 International License (CC-BY-NC-ND 4.0). Anyone may download, reuse, copy, reprint, or distribute the article without modifications or adaptations for non-profit purposes if they cite the original authors and source properly.

https://creativecommons.org/licenses/by-nc-nd/4.0/ 
Table 1. A comparison of the patient's characteristics and clinical course.

\begin{tabular}{|c|c|c|}
\hline & Chishiki et al. (2021) & Present case \\
\hline \multicolumn{3}{|l|}{ Maternal characteristics } \\
\hline Age (year) & 29 & 34 \\
\hline Chorioamnionitis & unknown & yes \\
\hline Cesarean delivery & yes & yes \\
\hline Detected microorganisms from vaginal culture & GBS & GBS \\
\hline \multicolumn{3}{|l|}{ Infant characteristics } \\
\hline Gestational age (weeks + days) & $39+4$ & $25+5$ \\
\hline Birth weight $(g)$ & 2,336 & 788 \\
\hline Gender & female & male \\
\hline Apgar score at $5 \mathrm{~min}$. & 9 & 6 \\
\hline Blood pressure (mmHg) & $85 / 59$ & $38 / 19$ \\
\hline Respiration rate (breaths/min) & 68 & 60 \\
\hline Heart rate (beats/min) & 162 & 160 \\
\hline Temperature $\left({ }^{\circ} \mathrm{C}\right)$ & 37.5 & 36.5 \\
\hline Leukocyte count $(/ \mu \mathrm{L})$ & 1,600 & 4,000 \\
\hline neutrophil (\%) & 52.5 & 61.2 \\
\hline Platelets count $\left(\times 10^{4} / \mu \mathrm{L}\right)$ & 29.2 & 15.4 \\
\hline C-reactive protein (mg/dL) & 6.19 & 1.40 \\
\hline Detected microorganisms from blood culture & GBS & GBS \\
\hline Detected microorganisms from CSF culture & GBS & not examined \\
\hline Type of cytokine/mediator removal therapy & ExTs & PMX-DHP \\
\hline Serum interleukin- $6(\mathrm{pg} / \mathrm{mL})$ before cytokine/mediator removal therapy & 86,900 & 6,792 \\
\hline Serum interleukin-6 (pg/mL) after cytokine/mediator removal therapy & 3,299 & 73 \\
\hline Frequency of cytokine/mediator removal therapy (times) & 2 & 2 \\
\hline Antibiotic administration (drug type) & ampicillin, cefotaxime & ampicillin, amikacin \\
\hline
\end{tabular}

GBS, group B streptococcus; CSF, cerebrospinal fluid; ExTs, exchange transfusions; PMX-DHP, Polymyxin B-immobilized fiber column for direct hemoperfusion.

\section{References}

Chishiki, M., Go, H., Endo, K., Katayama, N.U., Takehara, H. \& Namai, Y. (2021) Cytokine profiles before and after exchange transfusions in severe late-onset neonatal group B streptococcus meningitis: a case report. Tohoku. J. Exp. Med., 253, 269-273.

Goldstein, B., Giroir, B. \& Randolph, A.; Members of the International Consensus Conference on Pediatric Sepsis (2005) International pediatric sepsis consensus conference: definitions for sepsis and organ dysfunction in pediatrics. Pediatr. Crit. Care Med., 6, 2-8.

Nishizaki, N., Hara, T., Obinata, K., Nakagawa, M. \& Shimizu, T.
(2020) Clinical effects and outcomes after polymyxin B-immobilized fiber column direct hemoperfusion treatment for septic shock in preterm neonates. Pediatr. Crit. Care Med., 21, 156-163.

Nishizaki, N., Hirano, D., Miyasho, T., Obinata, K., Shoji, H. \& Shimizu, T. (2017) Evaluation of urinary IL-6 in neonates with septic shock treated with polymyxin B-immobilized fiber column. Pediatr. Int., 59, 1032-1033.

Nishizaki, N., Nakagawa, M., Hara, S., Oda, H., Kantake, M., Obinata, K., Uehara, Y., Hiramatsu, K. \& Shimizu, T. (2016) Effect of PMX-DHP for sepsis due to ESBL-producing E. coli in an extremely low-birthweight infant. Pediatr. Int., 58, 411-414 